\title{
DIMENSIONES DE LA SENSIBILIDAD A LA ANSIEDAD: EVIDENCIA CONFIRMATORIA DE LA ESTRUCTURA JERÁRQUICA
}

\author{
BONIFACIO SANDIN, PALOMA CHOROT, ROSA M. VALIENTE, \\ MIGUEL A. SANTED y LOURDES LOSTAO ${ }^{1}$ \\ Universidad Nacional de Educación a Distancia, Madrid \\ ${ }^{1}$ Universidad Pública de Navarra, Pamplona
}

(Aceptado en enero de 2004)

\begin{abstract}
Aunque el Índice de Sensibilidad a la Ansiedad (ASI; Reiss, Peterson, Gursky y McNally, 1986) es la escala más utilizada para la evaluación de la sensibilidad a la ansiedad (SA), su estructura aún no se conoce con precisión. En el presente estudio se analiza la estructura factorial de la versión española de la ASI con una muestra de 726 estudiantes universitarios (con un rango de edad de 18-34 años). Se ponen a prueba varios modelos alternativos, incluido un modelo factorial jerárquico. Los análisis factoriales exploratorios y confirmatorios indican que la ASI posee una estructura jerárquica compuesta por 3 factores primarios y 1 factor de orden superior; los factores primarios se corresponden con los referidos por Zinbarg, Barlow y Brown (1997) y Stewart, Taylor y Baker (1997): SA-somática, SA-cognitiva y SA-social. No existían diferencias entre grupos de sexo. Se discuten algunas posibles implicaciones para la psicopatología de los trastornos de ansiedad.
\end{abstract}

Palabras clave: Sensibilidad a la ansiedad, ASI, trastornos de ansiedad, análisis factorial confirmatorio, EQS, análisis factorial jerárquico.

\section{Dimensions of anxiety sensitivity: Confirmatory evidence for a hierarchic structure}

Although the Anxiety Sensitivity Index (ASI; Reiss, Peterson, Gursky \& McNally, 1986) is the most frequently used measure of the anxiety sensitivity construct, its dimensionality is yet a controversial issue. The present study examined the factor structure of the Spanish ASI in a sample of 726 university students (ranging in age from 18 to 34 years). The authors tested some current alternative models, including a hierarchical factor model. Exploratory and confirmatory factor analyses indicated a hierarchical factor structure consisting of 3 lower-order factors and 1 higher-order factor; the lowerorder factors were conceptually similar to the ones reported by Zinbarg. Barlow \& Brown (1997) and Stewart, Taylor \& Baker (1997), i.e., physical concerns, cognitive dyscontrol concerns, and social concerns. No differences were found between males and females. Some implications of these findings for psychopathology of anxiety disorders are discussed.

Key words: Anxiety sensitivity, ASI, anxiety disorders, confirmatory factor analysis, EQS, hierarchical factor analysis.

\section{INTRODUCCIÓN}

La sensibilidad a la ansiedad (SA) constituye uno de los nuevos conceptos más innovadores en el campo de los trastornos de ansiedad y, sin duda, uno de los

Correspondencia: Bonifacio Sandín, Universidad Nacional de Educación a Distancia, Facultad de Psicologia, Juan del Rosal 10. Ciudad Universitaria. 28040 Madrid. España. Correo-e: bsandin@psi.uned.es que más atención e interés han despertado a los investigadores y clínicos de este campo en los últimos años. La SA se define como el miedo a los síntomas de ansiedad, miedo que se supone debido a la creencia de que la ansiedad y sus sensaciones poseen consecuencias peligrosas o dañinas (Reiss y McNally, 1985). La SA no debe confundirse con el rasgo de ansiedad, tal y como ha sido demostrado 
empíricamente por algunos autores (Taylor, Koch, y Crockett, 1991; Sandín, Chorot y McNally, 2001). Muchas personas tienen un elevado rasgo de ansiedad (i.e., tienen tendencia a experimentar ansiedad ante cualquier situación estresante), pero sin experimentar miedo a los propios síntomas de ansiedad. La SA constituye el concepto central de la teoría de expectativa de la ansiedad (Reiss y McNally, 1985; Reiss, 1991), por cierto, una de las teorías más relevantes en el momento actual sobre la naturaleza, etiología y mantenimiento de los trastornos de ansiedad. En el marco de esta teoría, la SA ha sido conceptuada como un «miedo fundamental», en contraste con otros miedos no fundamentales (i.e., miedos derivados), como, por ejemplo, el miedo a volar en avión o el miedo a los perros. Si nos fijamos en estos dos últimos miedos, vemos que no existe una conexión lógica entre ellos: nadie dice que tiene miedo a volar porque teme a los perros, o viceversa. Sin embargo, si cabe afirmar que uno tiene miedo a volar, o a los perros, porque tiene miedo a los síntomas de ansiedad (p.ej., miedo a los síntomas de un posible ataque de pánico asociado a estas situaciones).

La SA fue propuesta como un factor de predisposición para el desarrollo de los trastornos de ansiedad (Reiss, 1991). La abundante investigación llevada a cabo sobre este constructo durante más de una década ha puesto de relieve que, si bien presenta una relación muy estrecha con los miedos y los trastornos de ansiedad en general (Valiente, Sandín y Chorot, 2002), su relación es mucho más específica con el trastorno de pánico (Taylor et al., 1991; Taylor, Koch, y McNally, 1992; Sandín, Chorot y McNally, 1996), siendo actualmente el principal factor de riesgo y marcador psicológico de este trastorno (McNally, 1994, 2002; Cox, Borger y Enns, 1999).
A pesar de la abundante literatura sobre la sensibilidad a la ansiedad, existe aún una marcada controversia sobre el número y naturaleza de sus posibles dimensiones. El estudio dimensional es un paso necesario en el proceso de la validación de constructo. Si bien se han llevado a cabo a este respecto múltiples estudios centrados en el principal instrumento de evaluación de la sensibilidad a la ansiedad, i.e., la Anxiety Sensitivity Index (ASI; Reiss, Peterson, Gursky y McNally, 1986), los resultados obtenidos han sido dispares, variando entre una estructura de un único factor (p.ej., Taylor et al., 1991, 1992; Sandín et al., 1996) y una estructura de cuatro factores (p.ej., Telch, Shermis y Lucas, 1989), si bien ha surgido una línea de evidencia más reciente a favor de una estructura jerárquica, constituida por tres factores de primer orden y un factor de orden superior (véase Zinbarg, Mohlman y Hong, 1999, para un análisis pormenorizado de este aspecto).

Zinbarg, Barlow y Brown (1997) y Stewart, Taylor y Baker (1997) publicaron los primeros trabajos, el primero con sujetos clínicos y el segundo con estudiantes universitarios, que sugerían una estructura de ASI consistente en tres factores primarios (denominados por Zinbarg et al. como Physical Concerns, Mental Incapacitation Concerns y Social Concerns). Resultados similares fueron obtenidos por Mohlman y Zinbarg (2000) con una muestra de personas de edad avanzada, y por Rodríguez et al. (2004) con una muestra de pacientes con trastornos de ansiedad. Más aun, en el estudio de Stewart et al. (1997) se concluyó que dicha estructura multifactorial y jerárquica era aproximadamente la misma en los grupos de varones y mujeres, a pesar de que entre ambos grupos existían diferencias en las puntuaciones de SA total y en el factor de miedo a síntomas físicos. 
La hipótesis sobre una estructura jerárquica de la ASI tiene importantes implicaciones de índole teórica y práctica. Desde el punto de vista teórico permite integrar gran parte de los datos aparentemente contradictorios sobre las dimensiones de la ASI (i.e., como una estructura unidimensional o multidimensional), siendo la hipótesis unifactorial congruente con la existencia del factor general de orden superior que predica el modelo jerárquico. Entre las implicaciones prácticas cabría mencionar la posibilidad de derivar varias subescalas de la ASI, correspondientes estas a los factores aislados.

Aunque el modelo jerárquico compuesto por tres factores primarios y un factor de orden superior ha sido replicado en algunos estudios (Zinbarg et al., 1999), estos proceden todos de población caucasiana (básicamente de Estados Unidos y Canadá). Más aun, algunos estudios recientes han puesto de relieve que esta estructura no se mantiene cuando se emplean ciertos tipos de población, como estudiantes afroamericanos (Carter, Miller, Sbrocco, Suchday y Lewis, 1999), puertorriqueños (Cintrón, Carter, Suchday, Sbrocco y Gray, en prensa) o indios americanos (Norton, De Coteau, Hope y Anderson, 2004), así como tampoco con muestras comunitarias (Schmidt y Joiner, 2002). Por tanto, resulta evidente que se precisan nuevos estudios sobre la estructura de la sensibilidad a la ansiedad, con objeto de determinar la consistencia del modelo jerárquico sugerido recientemente.

El presente trabajo tiene dos objetivos centrales. Mediante el primero de ellos pretendemos estudiar la estructura jerárquica de la ASI a partir de una muestra amplia de estudiantes universitarios españoles. Partiendo de los estudios iniciales basados en población de raza blanca (Estados Unidos y Canadá), hipotetizamos que la mejor solución factorial exploratoria debería consistir en tres factores primarios, relacionados con miedos a síntomas de tipo físico, de descontrol cognitivo (o incapacitación mental) y de tipo social, respectivamente. Además, hipotetizamos que estos tres factores de orden inferior deberían saturar en un factor de segundo orden, lo cual sería consistente con el modelo jerárquico de la sensibilidad a la ansiedad. Tal estructura jerárquica sólo ha sido probada mediante análisis factorial confirmatorio por Zinbarg et al. (1997) en una muestra de sujetos clínicos; que nosotros sepamos, aún no se ha demostrado esta estructura de la ASI con este método de análisis en muestras de participantes no clínicos. En segundo lugar, y partiendo de que se han sugerido diferencias sexuales en los niveles de sensibilidad a la ansiedad, pretendemos llevar a cabo análisis factoriales exploratorios para estudiar las posibles diferencias entre varones y mujeres en la configuración factorial relativa a los factores primarios y al factor de orden superior. De acuerdo con el estudio de Stewart et al. (1997), nuestra hipótesis no defiende que existan diferencias sexuales a este respecto, sugiriéndose la existencia de invarianza factorial entre los grupos de varones y mujeres.

\section{MÉTODO}

\section{Participantes}

Los participantes consistieron en una muestra total de 726 estudiantes universitarios pertenecientes a universidades de Madrid y Pamplona. La muestra estaba constituida por 523 mujeres (72\%) y 203 varones (28\%). Ambos grupos poseían medias de edad similares; la media de edad de las mujeres era de 21,0 $(D T=2,6)$; la media de edad del grupo de hombres era de 21,2 $(D T=2,5)$. El rango de edad para la muestra total fue de 18 a 34 años. 


\section{Pruebas psicológicas}

Los participantes cumplimentaron de forma colectiva (en grupos de 20-50 alumnos) en diversos centros docentes universitarios de Madrid y Pamplona la siguiente escala (junto a otros cuestionarios):

\section{Índice de Sensibilidad a la Ansiedad} [Anxiety Sensitivity Index, ASI] (Peterson y Reiss, 1992). Se aplicó la versión española de Sandín y Chorot (Sandín, Valiente y Chorot, 1999; véase el Anexo I). Al igual que la versión original, consta de 16 items y mantiene su misma estructura general y el mismo formato de respuesta. El participante debe indicar el grado en que experimenta cada uno de los 16 enunciados según una escala tipo Likert, pudiendo variar entre "Nada en absoluto" (0) y "Muchísimo" (4). Los items hacen referencia a reacciones de miedo/ansiedad ante la experiencia de síntomas de tipo somático (p.ej., «Me asusto cuando mi corazón late de forma rápida»), síntomas observables socialmente («Para mi es importante no dar la impresión de estar nervioso"), y síntomas de descontrol cognitivo (p.ej., "Me asusto cuando soy incapaz de mantener mi mente concentrada en una tarea"). En anteriores publicaciones sobre la ASI hemos puesto de relieve que esta prueba posee excelentes propiedades psicométricas, tanto con población clínica como no clínica (Sandín et al., 1996, 2001).

\section{RESULTADOS}

\section{Análisis factorial de la estructura de orden inferior de la ASI}

Para analizar la estructura factorial de la ASI llevamos a cabo análisis factoriales exploratorios, mediante análisis factoriales de ejes principales. Para la extracción del número de factores se tuvieron en cuenta los siguientes criterios teóricos y/o empíricos: (1) Modelo teórico sobre la estructura jerárquica de la ASI. (2) Evidencia de la literatura sobre la consistencia de una estructura unidimensional o multifactorial de la ASI. (3) Criterio de Kaiser; se basa en la retención de factores con autovalores por encima de la unidad. (4) Test de scree; se basa en la representación gráfica de todos los autovalores (Cattell, 1966). (5) Finalmente, se aplicó el criterio de interpretabilidad de las diferentes estructuras factoriales factibles (Gorsuch, 1983). En los modelos multifactoriales, y de acuerdo con la consistencia de la literatura sobre las correlaciones entre los factores primarios, se empleó la rotación oblicua oblimin (este es el método de rotación más aceptado para los análisis factoriales exploratorios). Los análisis factoriales se aplicaron de forma separada para la muestra total, para el grupo de varones, y para el grupo de mujeres.

Los resultados de estos análisis se indican en la Tabla 1. Como puede apreciarse en dicha tabla, obtuvimos tres factores primarios para las cuatro condiciones de análisis (muestra total, varones, y mujeres). Los porcentajes de varianza iniciales explicados por los tres factores eran muy similares en los tres análisis $(49,2 \%$, $51,2 \%$ y $49,1 \%$, respectivamente). También puede apreciarse que el significado conceptual de los factores aislados es idéntico en varones, mujeres y en la muestra total. El primer factor (Factor 1) está configurado por los Items 9, 10, 6, 8, 4,11 y 14, y lo denominamos Factor Somático ya que las saturaciones relevantes en este factor corresponden a los items de la ASI que representan miedos a síntomas somáticos. El segundo factor (Factor 2) está definido por cinco items de la ASI $(15,16,2,12$ y 3$)$, y es denominado Factor Cognitivo, ya que estos se refieren a reacciones de sensibilidad a la ansiedad 
Tabla 1. Análisis factorial de ejes principales de la ASI: Pesos factoriales tras rotación oblicua (oblimin) para la muestra total y para los grupos de varones y mujeres

\begin{tabular}{|c|c|c|c|c|c|c|c|c|c|}
\hline \multirow[b]{2}{*}{ Item ASI (resumido*) } & \multicolumn{3}{|c|}{ Total $(N=726)$} & \multicolumn{3}{|c|}{ Varones $(N=203)$} & \multicolumn{3}{|c|}{ Mujeres $(N=523)$} \\
\hline & $F 1$ & $F 2$ & $F 3$ & $F 1$ & $F 2$ & $F 3$ & $F 1$ & $F 2$ & $F 3$ \\
\hline 9. Preocupación ataque cardíaco & $\mathbf{0 , 8 1}$ & $-0,01$ & $-0,17$ & $\mathbf{0 , 7 9}$ & $-0,11$ & 0,03 & $\mathbf{0 , 8 6}$ & 0,05 & $-0,22$ \\
\hline 10. Respirar con dificultad & $\mathbf{0 , 7 6}$ & $-0,06$ & 0,02 & $\mathbf{0 , 7 8}$ & $-0,04$ & $-0,02$ & $\mathbf{0 , 7 0}$ & $-0,05$ & 0,11 \\
\hline 6. Taquicardia & $\mathbf{0 , 7 3}$ & 0,01 & 0.01 & 0,71 & 0,06 & $-0,02$ & 0,69 & $-0,01$ & 0,10 \\
\hline 8. Náuseas & $\mathbf{0 , 5 9}$ & 0,02 & 0,13 & 0,57 & 0,17 & 0,05 & $\mathbf{0 , 5 3}$ & $-0,03$ & 0,25 \\
\hline 4. Desmayo o mareo & $\mathbf{0 , 5 1}$ & 0,01 & 0,15 & 0,51 & 0,03 & $-0,02$ & 0,44 & 0,00 & 0,34 \\
\hline 11. Preocupación estómago enfermo & $\mathbf{0 , 5 0}$ & 0,14 & $-0,05$ & $\mathbf{0 , 3 5}$ & 0,29 & 0,07 & $\mathbf{0 , 5 6}$ & 0,13 & $-0,11$ \\
\hline 14. Sensaciones corporales & $\mathbf{0 , 3 8}$ & 0,26 & $-0,06$ & 0,25 & $\mathbf{0 , 3 4}$ & $-0,02$ & $\mathbf{0 , 4 0}$ & 0,23 & $-0,01$ \\
\hline 15. Preocupación estar nervioso & $-0,02$ & $\mathbf{0 , 8 4}$ & $-0,17$ & $-0,02$ & 0,76 & $-0,07$ & 0,04 & 0,88 & $-0,20$ \\
\hline 16. Estar nervioso & 0,06 & $\mathbf{0 , 7 0}$ & 0,00 & 0,08 & $\mathbf{0 , 5 9}$ & 0,17 & 0,07 & 0,71 & 0,05 \\
\hline 2. Preocupación volverse loco & $-0,01$ & 0,55 & 0,04 & $-0,01$ & 0,75 & $-0,12$ & 0,00 & 0,48 & 0,09 \\
\hline 12. Incapacidad concentración & 0,09 & $\mathbf{0 , 5 4}$ & 0,06 & 0,06 & $\mathbf{0 , 5 6}$ & 0,07 & 0,10 & $\mathbf{0 , 5 0}$ & 0,11 \\
\hline 3. Me asusto si tiemblo & 0,19 & $\mathbf{0 , 3 2}$ & 0,25 & 0,08 & $\mathbf{0 , 5 8}$ & 0,01 & 0,15 & 0,23 & $\mathbf{0 , 4 4}$ \\
\hline 13. Otros notan cuando tiemblo & $-0,03$ & $\mathbf{0 , 3 3}$ & 0,21 & $-0,08$ & $\mathbf{0 , 4 1}$ & 0,11 & -0.06 & 0,30 & 0,30 \\
\hline 1. Preocupación no parecer nervioso & $-0,02$ & 0,04 & $\mathbf{0 , 4 9}$ & $-0,12$ & $-0,01$ & $\mathbf{0 , 8 3}$ & $-0,01$ & 0,04 & 0,39 \\
\hline 5. Preocupación controlar emociones & 0,06 & 0.01 & 0,43 & 0,10 & 0.06 & 0,45 & 0,10 & 0,02 & 0,31 \\
\hline 7. Embarazoso estómago hace ruidos & 0,29 & 0,03 & $\mathbf{0 , 3 1}$ & 0,32 & 0,04 & 0,23 & 0,24 & $-0,05$ & $\mathbf{0 , 3 1}$ \\
\hline Varianza explicada (\%) & 31,0 & 10,2 & 8,0 & 33,3 & 10.0 & 7,9 & 30,3 & 10,4 & 8,4 \\
\hline
\end{tabular}

Nota: F1 = Factor somático (Miedo a síntomas somáticos), F2 = Factor cognitivo (Miedo al descontrol cognitivo), F3 = Factor social (Miedo a síntomas observables públicamente). Los pesos factoriales $\geq 0,30$ se indican en negrita. * Véase el Anexo I para la descripción completa de los items.

relacionadas con la preocupación o miedo al descontrol cognitivo. Finalmente, el tercer factor (Factor 3), o Factor Social, se refiere a la dimensión social de la sensibilidad a la ansiedad (preocupación por reacciones observables públicamente). Las correlaciones entre los factores variaron entre 0,31 y 0,40 (muestra total), entre 0,35 y 0,52 (varones), y entre 0,32 y 0,40 (mujeres). En los tres casos, las mayores correlaciones se dieron entre los Factores 1 y 2, y las menores entre los Factores 2 y 3 .

Se aprecia que el factor más consistente es el que representa la dimensión somática de la SA (i.e., miedo a síntomas somáticos de ansiedad), correspondiendo el menos consistente al factor o dimensión social de la SA (reacciones observables públicamente). Para el grupo de varones, el Ítem 13 ( «Cuando siento que tiemblo la gente suele darse cuenta"), en contra de lo esperado, presenta un peso más relevante en el Factor Cognitivo que en el Factor Social, mientras que en el grupo de mujeres satura por igual en ambos factores. Aunque hemos asimilado este ítem a la dimensión social basándonos en su contenido descriptivo, sin embargo, los datos empíricos de estos análisis sugieren una relación más estrecha con el temor al descontrol cognitivo (Factor Cognitivo) que a la SA social. Respecto al Ítem 3 ( «Me asusto cuando siento que tiemblo"), si bien es concebido como un ítem representativo de la dimensión cognitiva, puede observarse que para el grupo de mujeres parece representar mejor la dimensión social que la cognitiva (véase la Tabla 1). Aparte de los Items 13 y 3 , Otros items que resultan parcialmente inconsistentes son los Items 7 y 14. El Ítem 7 ( Me resulta embarazoso o molesto cuando mi estómago hace ruidos»), para el grupo de varones, presenta la máxima saturación 
en el Factor Somático, y no en el Factor Social como era esperable. Así mismo, el Ítem 14 («Las sensaciones corporales poco habituales (raras) me asustan»), para el grupo e varones, satura más en el Factor Cognitivo que en el Factor Somático. Se observa pues, que en los varones algunos items de la ASI pueden tener un significado de temor al descontrol cognitivo más de lo esperado, fenómeno que en las mujeres de produce más bien en relación con el temor o preocupación a las reacciones de ansiedad observables públicamente.

Dado que en la literatura también se ha venido sugiriendo la naturaleza unidimensional de la ASI (Reiss et al., 1986; Taylor et al., 1991, 1992; Sandín et al., 1996), examinamos así mismo la estructura factorial extrayendo un único factor. En los tres análisis efectuados (muestra total, varones, y mujeres) se obtuvieron resultados similares. Aparte de que los procedimientos empleados para determinar el número de factores sugerían la estructura de tres factores mejor que la de un factor, el análisis de ejes principales, con extracción de un factor, reveló que la estructura unidimensional era menos apropiada que la tridimensional al menos por los dos hechos siguientes observados en la primera: (a) las comunalidades eran muy bajas para algunos items, especialmente para los relativos a la sensibilidad a la ansiedad social, y (b) existían algunas saturaciones menores de 0,30 (aunque no se incluyen estos análisis, pueden ser facilitados solicitándolos al primer autor).

Análisis factorial de la estructura de orden superior de la ASI

El análisis de la estructura de orden superior de la ASI se llevó a cabo factorizando (ejes principales) las puntuaciones factoriales de los tres factores primarios rotados oblicuamente, para las tres muestras de datos (total, varones y mujeres). Los resultados de estos análisis se indican en la Tabla 2. La principal conclusión que se obtiene de los tres análisis efectuados es que, en los tres casos, los resultados apoyan claramente la existencia de un único factor de orden superior. Por tanto, estos datos sugieren que la ASI posee una estructura jerárquica, la cual podría consistir en un factor general de orden superior y tres factores primarios de orden inferior. Para examinar esta posible estructura jerárquica, analizamos la varianza de los items atribuible al factor general y la varianza residualizada (varianza específica atribuible a cada factor primario) (véase el epígrafe siguiente).

\section{Análisis jerárquico de la estructura factorial de la ASI}

Para llevar a cabo el análisis jerárquico de la ASI se aplicó la transformación de Schmid-Leiman (1957) para descompo-

Tabla 2. Análisis factorial de ejes principales de la estructura jerárquica de la ASI: Pesos factoriales en el factor de orden superior para la muestra total y para los grupos de varones y mujeres

\begin{tabular}{lccc} 
Factores primarios rotados (puntuaciones factoriales) & $\begin{array}{c}\text { Total } \\
(N=726)\end{array}$ & $\begin{array}{c}\text { Varones } \\
(N=203)\end{array}$ & $\begin{array}{c}\text { Mujeres } \\
(N=523)\end{array}$ \\
\hline Miedo a síntomas somáticos (Somático) & 0,81 & 0,78 & 0,76 \\
Miedo al descontrol cognitivo (Cognitivo) & 0,72 & 0,79 & 0,63 \\
Miedo a síntomas observables públicamente (Social) & 0,60 & 0,56 & 0,65 \\
Varianza explicada (\%) & 51,1 & 51,5 & 46,6 \\
\hline
\end{tabular}


Tabla 3. Análisis jerárquico de la ASI: Pesos factoriales en el factor general ( $g$ ) y pesos residualizados para la muestra total y para los grupos de varones y mujeres, tras aplicarse la transformación de Schmid-Leiman

\begin{tabular}{|c|c|c|c|c|c|c|c|c|c|c|c|c|}
\hline \multirow[b]{2}{*}{ Ítem ASI (resumido) } & \multicolumn{4}{|c|}{ Total $(N=726)$} & \multicolumn{4}{|c|}{ Varones $(N=203)$} & \multicolumn{4}{|c|}{ Mujeres $(N=523)$} \\
\hline & $g$ & $F 1$ & $F 2$ & $F 3$ & $g$ & $F 1$ & $F 2$ & $F 3$ & $g$ & $F 1$ & $F 2$ & $F 3$ \\
\hline 9. Preocupación ataque cardíaco & $\mathbf{0 , 5 5}$ & 0,48 & $-0,01$ & $-0,14$ & $\mathbf{0 , 5 7}$ & $\mathbf{0 , 4 9}$ & $-0,07$ & 0,02 & 0,54 & $\mathbf{0 , 5 6}$ & $0,04-$ & $-0,1$ \\
\hline 10. Respirar con dificultad & $\mathbf{0 , 5 8}$ & 0,45 & $-0,04$ & 0,02 & 0,57 & 0,48 & $-0,02$ & $-0,02$ & 0,57 & 0,46 & -0.04 & 0,08 \\
\hline 6. Taquicardia & $\mathbf{0 , 6 0}$ & $\mathbf{0 , 4 3}$ & 0,01 & 0,01 & $\mathbf{0 , 5 9}$ & 0,44 & 0,04 & $-0,02$ & $\mathbf{0 , 5 8}$ & 0,45 & $-0,01$ & 0,08 \\
\hline 8. Náuseas & $\mathbf{0 , 5 7}$ & $\mathbf{0 , 3 5}$ & 0,01 & 0,11 & 0,61 & 0,35 & 0,10 & 0,04 & 0,55 & 0,35 & $-0,02$ & 0,19 \\
\hline 4. Desmayo o mareo & $\mathbf{0 , 5 1}$ & $\mathbf{0 , 3 0}$ & 0,01 & 0,12 & $0, \mathbf{4 1}$ & $\mathbf{0 , 3 2}$ & 0,02 & $-0,02$ & $\mathbf{0 , 5 5}$ & 0,29 & 0,00 & 0,26 \\
\hline 11. Preocupación estómago enfermo & $\mathbf{0 , 4 8}$ & $\mathbf{0 , 3 0}$ & 0,10 & $-0,04$ & $\mathbf{0 , 5 4}$ & 0,22 & 0,18 & 0,06 & $\mathbf{0 , 4 4}$ & 0,36 & $0,08-$ & $-0,08$ \\
\hline 14. Sensaciones corporales & 0,46 & 0,23 & 0,18 & $-0,05$ & 0,46 & 0,16 & 0,21 & $-0,02$ & 0,44 & 0,26 & $0,18-$ & $-0,01$ \\
\hline 15. Preocupación estar nervi & $\mathbf{0 , 5 5}$ & $-0,01$ & $\mathbf{0 , 5 8}$ & $-0,14$ & $\mathbf{0 , 5 5}-$ & $-0,01$ & 0,46 & $-0,06$ & $\mathbf{0 , 4 5}$ & 0,03 & $\mathbf{0 , 6 8 -}$ & $-0,15$ \\
\hline 16. Estar nervioso & 0,55 & 0,04 & 0,48 & 0,00 & 0,62 & 0,05 & $\mathbf{0 , 3 6}$ & 0,14 & $\mathbf{0 , 5 3}$ & 0,05 & $\mathbf{0 , 5 5}$ & 0,04 \\
\hline 2. Preocupación volverse loco & $\mathbf{0 , 4 1}$ & $-0,01$ & $\mathbf{0 , 3 8}$ & 0,03 & $\mathbf{0 , 5 2}$ - & $-0,01$ & 0,46 & $-0,10$ & $\mathbf{0 , 3 6}$ & 0,00 & $\mathbf{0 , 3 7}$ & 0,07 \\
\hline 12. Incapacidad concentración & $\mathbf{0 , 5 0}$ & 0,05 & 0,37 & 0,05 & $\mathbf{0 , 5 3}$ & 0,04 & $\mathbf{0 , 3 4}$ & 0,06 & $\mathbf{0 , 4 6}$ & 0,07 & $\mathbf{0 , 3 9}$ & 0,08 \\
\hline 3. Me asusto si tiemblo & $\mathbf{0 , 5 3}$ & 0,11 & 0,22 & 0,20 & $\mathbf{0 , 5 2}$ & 0,05 & $\mathbf{0 , 3 5}$ & 0,00 & $\mathbf{0 , 5 4}$ & 0,10 & 0,1 & $\mathbf{0 , 3 4}$ \\
\hline 13. Otr & $\mathbf{0 , 3 4}$ & $-0,02$ & 0,23 & 0,17 & $\mathbf{0 , 3 2}$ & $-0,05$ & 0,25 & 0,09 & 0,3 & $-0,04$ & 0,2 & 0,22 \\
\hline 1. Preocupación $r$ & $\mathbf{0 , 3 0}$ & $-0,01$ & 0,03 & $\mathbf{0 , 3 9}$ & $\mathbf{0 , 3 6}$ & $-0,0$ & $-0,01$ & 0,69 & 0,2 & $-0,01$ & 0,03 & $\mathbf{0 , 3 0}$ \\
\hline 5. Preocupación controlar & $\mathbf{0 , 3 1}$ & 0,04 & 0,01 & $\mathbf{0 , 3 5}$ & 0,38 & 0,06 & 0,04 & 0,37 & 0,29 & 0,07 & 0,02 & 0.24 \\
\hline 7. Embarazoso estómago hace ruidos & 0,44 & 0,17 & 0,02 & 0,25 & $\mathbf{0 , 4 1}$ & 0,20 & 0,02 & 0,19 & $\mathbf{0 , 3 5}$ & 0,16 & $-0,04$ & 0,24 \\
\hline Varianza explicada (\%) & 23,9 & 6,5 & 6,2 & 3,0 & 25,6 & 6,4 & 5,9 & 4,4 & 21,6 & 7.4 & 7.4 & 3,5 \\
\hline
\end{tabular}

Nota: $\mathrm{g}=$ Factor general, F1 = Factor somático (Miedo a síntomas somáticos), F2 = Factor cognitivo (Miedo al descontrol cognitivo), F3 = Factor social (Miedo a síntomas observables públicamente). Los pesos factoriales $\geq$ 0,30 se indican en negrita. * Véase el Anexo I para la descripción completa de los items.

ner las varianzas correspondientes al factor general y a los factores de orden inferior. Mediante este procedimiento determinamos para cada ítem de la ASI la saturación en el factor general ( $g$ ) y las saturaciones residualizadas en los tres factores de orden inferior. Los pesos residualizados representan la contribución única de cada factor de orden inferior, una vez que se ha eliminado la contribución del factor general. El método de Schmid-Leiman resulta particularmente importante ya que permite separar la varianza de los items explicada por el factor general de la varianza explicada por los factores primarios (y no explicada por el factor general).

En la Tabla 3 exponemos los resultados obtenidos tras los análisis jerárquicos de la ASI realizados de forma separada para las tres condiciones (muestra total, varones y mujeres). Puede observarse que se mantiene un patrón similar en los tres análisis efectuados. En los tres casos se dan saturaciones relevantes de prácticamente todos los items en el factor general, y de grupos aislados de items en los factores de orden inferior. Estas saturaciones son consistentes con la hipótesis jerárquica, la cual supone que mientras todos los items deben saturar en el factor general, en cada factor primario sólo deberían saturar los items relevantes al factor específico. En la tabla también puede observarse que la varianza explicada por el factor general es superior a la varianza acumulada explicada por los tres factores primarios, lo que sugiere que la varianza de la ASI está más determinada por el factor general común a todos los items que por los componentes específicos propios de cada factor de orden inferior. Una vez más, este patrón no difiere entre varones y mujeres. Final- 
mente, en relación con la caracterización de los tres factores primarios descritos atrás (véase la Tabla 1), el factor social resulta ser menos consistente (véanse las saturaciones en este factor de los items (4) relevantes al mismo; Tabla 3 ).

\section{Análisis factorial confirmatorio}

Aunque nuestros análisis factoriales exploratorios sugieren que la ASI posee una estructura jerárquica constituida por un factor general y tres factores de orden inferior, existe aún bastante controversia sobre este aspecto (véase Taylor, 1995a; Zinbarg et al., 1999). Entre las diversas alternativas a la estructura de tres dimensiones primarias y, aparte de la estructura unidimensional ya comentada, merece la pena resaltar la estructura de dos factores sugerida en algunos estudios recientes sobre la SA (Sandín, Chorot, Santed y Valiente, 2002; Zvolensky et al., 2003; Cintrón et al., en prensa). Por estas razones, nos pareció necesario llevar a cabo análisis factoriales confirmatorios con objeto de probar cuál de los cuatro principales modelos competitivos (1 factor; 2 factores; 3 factores; y 3 factores y uno de orden superior-modelo jerárquico) era más consistente con los datos empíricos (para los modelos de 2 y 3 factores se incluyó la variante oblicuo vs. ortogonal, por lo que en total se evaluaron 6 modelos). Para la realización de los análisis confirmatorios nos basamos en la muestra total y se empleó el programa de ecuaciones estructurales EQS (Bentler, 1995). Se contrastaron los seis modelos indicados, que varían en función del número de factores, en el carácter oblicuo u ortogonal, y en la ordenación jerárquica o no jerárquica. La identificación del modelo de tres factores y del modelo jerárquico se efectuó partiendo de nuestros datos exploratorios previos, los cuales coinciden (excepto para el Ítem 3) con los indi- cados por Zinbarg et al. (1997) y Stewart et al. (1997) (véase la Tabla 1). La identificación del modelo de dos factores se realizó a partir de los datos de Sandín et al. (2002) y Zvolensky et al. (2003), según los cuales existe una dimensión común de tipo social-cognitiva (i.e., el modelo consistiría en un factor de tipo somático y un segundo factor determinado por los items referidos al descontrol cognitivo y a las reacciones observables de ansiedad); este modelo guarda cierta semejanza con el propuesto recientemente por Cintrón et al. (en prensa).

Utilizamos modelos congenéricos de análisis factorial confirmatorio. Las varianzas de las variables latentes se fijaron en 1,0. Las varianzas de los términos de error fueron especificadas como parámetros libres. No se permitió la covariación entre los residuos. Como punto de partida, las saturaciones de las variables se estimaron en 1,0 para el factor relevante y en 0,0 para el factor no relevante. Se empleó el método de estimación de máxima verosimilitud robusto (ML-robusto). Este método permite corregir los posibles errores debidos a la distribución no normal de los datos. Usamos los siguientes indicadores de bondad de ajuste: (1) chi-cuadrado dividido por los grados de libertad (cocientes $\leq 5,0$ indican buen ajuste; a menor índice mejor ajuste), (2) índice de ajuste comparativo de Bentler (RCFI, Robust comparative fit index), (3) índice de bondad de ajuste LISREL (GFI, goodness of fit index), (4) raíz media cuadrática residual (RMR, root mean square residual), (5) raíz media cuadrática del error de aproximación (RMSEA, root mean square error of approximation), $y$ (6) criterio de información de Akaike (AIC, Akaike's information criterion). Para que exista un buen ajuste, el RCFI y el GFI deberían acercarse al valor de 0,90 - (cuanto mayor sea el valor, mejor ajuste), y los valores RMR y RMSEA al menos deberían ser $\leq 0,08$ (cuanto menor sea el 
Tabla 4. Índices de bondad de ajuste para los diferentes modelos relacionados con la estructura de la ASI, basados en los análisis factoriales confirmatorios EQS $(N=726)$

\begin{tabular}{lcccccc}
\hline Modelo estructural de la ASI & $\chi^{2} / g l$ & $R C F I$ & $G F I$ & $R M R$ & $R M S E A$ & $A I C$ \\
\hline Un factor (modelo unidimensional) & 7,36 & 0,75 & $\mathbf{0 , 8 3}$ & 0,08 & 0,11 & 784,0 \\
Dos factores ortogonales & 6,92 & $\mathbf{0 , 7 6}$ & $\mathbf{0 , 8 7}$ & 0,18 & $\mathbf{0 , 1 0}$ & 680,8 \\
Dos factores correlacionados & $\mathbf{5 , 0 7}$ & $\mathbf{0 , 8 4}$ & $\mathbf{0 , 8 9}$ & 0,07 & $\mathbf{0 , 0 8}$ & $\mathbf{4 5 2 , 1}$ \\
Tres factores ortogonales & 7,38 & $\mathbf{0 , 7 4}$ & $\mathbf{0 , 8 6}$ & 0,18 & $\mathbf{0 , 1 1}$ & $\mathbf{7 3 5 , 4}$ \\
Tres factores correlacionados & 4,76 & $\mathbf{0 , 8 5}$ & 0,90 & 0,06 & $\mathbf{0 , 0 8}$ & $\mathbf{4 0 4 , 8}$ \\
Tres factores y un factor de orden superior (modelo jerárquico) & 3,54 & $\mathbf{0 , 9 1}$ & 0,91 & 0,06 & $\mathbf{0 , 0 8}$ & $\mathbf{4 1 6 , 8}$ \\
\hline
\end{tabular}

Nota: RCFI = robust comparative fit index; GFI = LISREL goodness of fit index; RMR = root mean square residual; RMSEA = root mean square error of approximation; AIC = Akaike's information criterion.

valor, mejor ajuste). El AIC es un indicador relativo; en ausencia de otros criterios definitorios, el mejor modelo es el que posea un AIC más bajo.

Los índices de bondad de ajuste relativos a los 6 modelos que fueron contrastados empíricamente están indicados en la Tabla 4. Tras la inspección de la tabla puede observarse que (1) los modelos multifactoriales se ajustan mejor que el modelo unifactorial, (2) los modelos correlacionales se ajustan mejor que los ortogonales, y (3) los modelos correlacionales de tres factores se ajustan mejor que el bifactorial. En primer lugar, estos datos confirman la hipótesis de que la ASI representa varias dimensiones, estando estas correlacionadas entre sí (Taylor, 1995b; Cox, Parker y Swinson, 1996). Las correlaciones entre los factores (modelos de 2 y 3 factores) fueron moderadas/altas (variaron entre 0,56 y 0,64 ), lo cual sugería un cierto nivel de solapamiento y una justificación del factor general. Si comparamos los índices de bondad de ajuste relativos a los dos modelos de tres factores se aprecia que, aun teniendo ambos buenos niveles de ajuste, el modelo jerárquico resulta ser ligeramente superior (con la pequeña salvedad de que el AIC es algo inferior en el no jerárquico). Este resultado es consistente con los datos (Zinbarg et al., 1997; Mohlman y Zinbarg, 2000) y con el análisis de la literatura (Zinbarg et al., 1999) sobre la estructura jerárquica de la ASI.
En la Figura 1 representamos el modelo jerárquico sobre la SA basado en los resultados de nuestro análisis estructural. Como puede observarse, en el modelo jerárquico existe un factor general que se relaciona con los tres factores primarios y, a su vez, estos últimos se relacionan de forma específica con los 3 grupos de items de la ASI. Se indican las estimaciones paramétricas en forma de coeficientes completamente estandarizados (todos son significativos estadísticamente). Los coeficientes denotan los efectos del factor general (que denominamos sensibilidad a la ansiedad, SA) sobre los tres factores de orden inferior (SA-Somática, SA-Cognitiva y SA-Social), y los efectos de estos sobre las variables observadas relevantes a cada factor. Puede observarse que el efecto del factor general es elevado sobre las dimensiones somática y cognitiva de la sensibilidad a la ansiedad, y moderado sobre la dimensión social, lo que indica que explica una menor proporción de la varianza de este factor. Así mismo, puede observarse que los efectos del factor SA-Social sobre las variables observadas son más bajos que los efectos de los factores SA-Somática y SA-Cognitiva. No obstante, tomado el modelo en conjunto, los elevados a moderados valores de los coeficientes indican que el modelo es bastante robusto, y sugieren que la SA posee una estructura claramente multidimensional $\mathrm{y}$ jerárquica. 


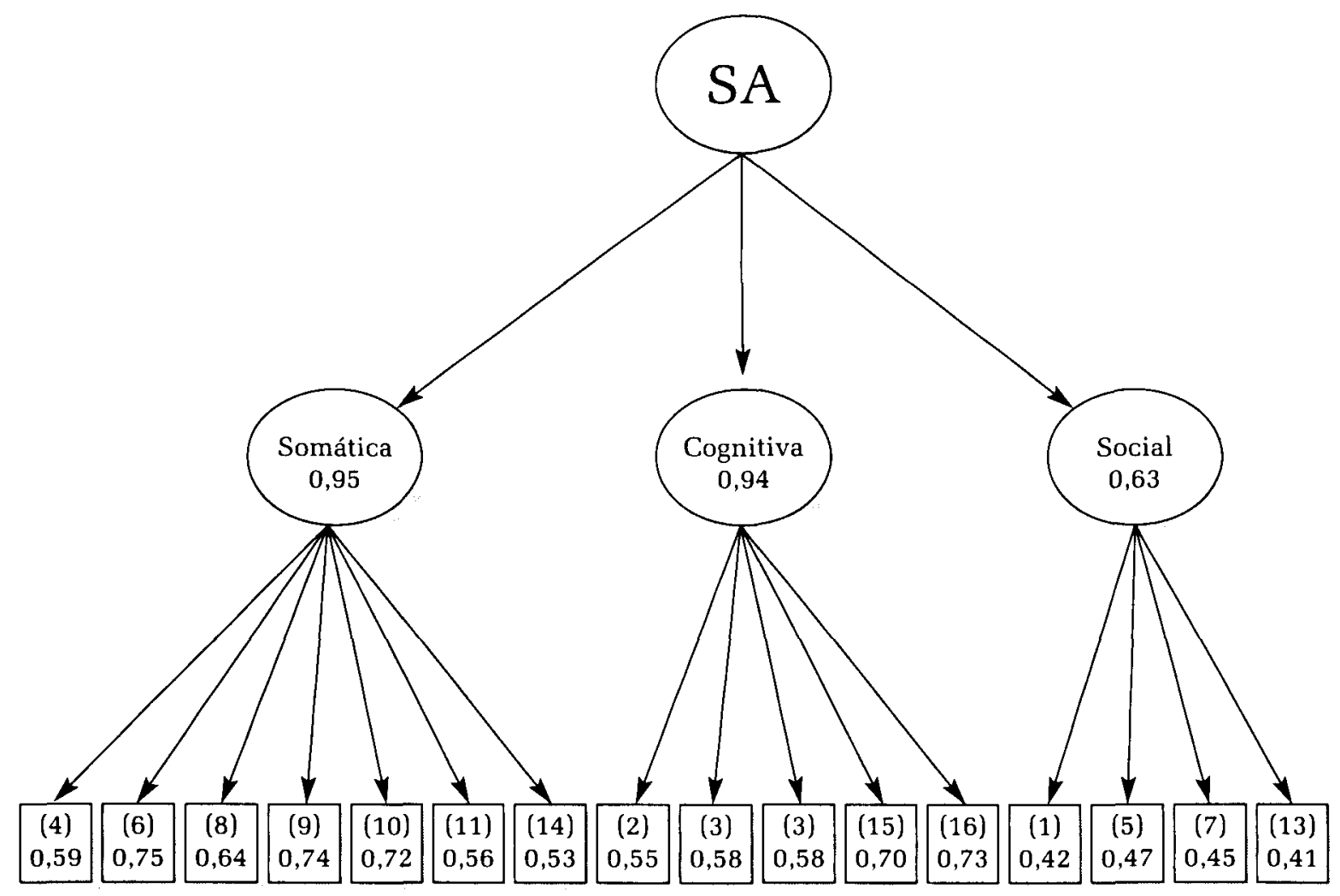

Figura 1. Modelo estructural de la sensibilidad a la ansiedad (SA). Se indican los efectos (coeficientes estandarizados) de las variables independientes sobre las dependientes. Las variables latentes se representan en círculos; las variables observadas se representan en rectángulos (i.e., items de la ASI; entre paréntesis se indica el número de ítem). Para mayor explicación véase el texto.

\section{DISCUSIÓN}

Los resultados del presente estudio apoyan mediante análisis factorial confirmatorio la estructura jerárquica de la SA, consistente esta en un factor general y tres factores de orden inferior que denominamos SA-Somática (miedo a los síntomas físicos de ansiedad), SA-Cognitiva (miedo al descontrol cognitivo) y SASocial (miedo a las reacciones observables de ansiedad). Estos datos son coherentes con algunos estudios previos sobre la estructura de la SA (Taylor, 1995a, 1995b; Cox et al., 1996; véase Zinbarg et al., 1999), y en particular con los resultados más recientes de Zinbarg et al. (1997), Stewart et al. (1997), Mohlman y Zinbarg (2000) y Rodríguez et al. (2004).
Al igual que el estudio de Stewart et al. (1997), los resultados de este trabajo indican que no existen diferencias sustanciales entre varones y mujeres en la estructura de la sensibilidad a la ansiedad.

Nuestros datos constituyen la primera evidencia empírica basada en análisis factorial confirmatorio sobre dicha estructura jerárquica en muestras de participantes no clínicos. Dos estudios con pacientes ansiosos (Zinbarg et al., $1997 \mathrm{y}$ Rodríguez et al., 2004) también constataron mediante análisis factorial confirmatorio la validez del modelo jerárquico aquí presentado. En los estudios de Stewart et al. (1997), con estudiantes universitarios, y Mohlman y Zinbarg (2000), con participantes de edad avanzada, también probaron la validez del modelo 
jerárquico aquí presentado, aunque sin aplicar métodos de análisis estructural (i.e., análisis factorial confirmatorio) (Stewart et al., únicamente emplearon la transformación de Schmid-Leiman, y Mohlman y Zinbarg utilizaron un procedimiento exploratorio poco común que denominan «representación bifactorial», el cual no está exento de dudas sobre su validez).

Podría afirmarse que, en términos generales, los resultados del presente estudio indican que existe una convergencia transcultural en la estructura de la ASI para la población de raza blanca (población española y población de Estados Unidos y Canadá). Sin embargo, tales resultados contrastan con los obtenidos a partir de otros grupos de población, tales como los pertenecientes a muestras comunitarias (Schmidt y Joiner, 2002), estudiantes universitarios afroamericanos (Carter et al., 1999), indios de América del Norte (Norton et al., 2004), nativos de Puerto Rico (Cintrón et al., en prensa). En ninguno de estos tres estudios se obtiene la estructura propuesta en el presente trabajo (Carter et al. obtienen 4 factores: cognitivo, social, sensaciones corporales, y síntomas cardiovasculares; Norton et al. obtienen un solo factor; y Cintrón et al. obtienen 2 factores: sensibilidad a la ansiedad, y emocional). En cierto modo nos ha sorprendido que nuestros datos no coincidan con los de Cintrón et al., ya que, en último término, la población que participó en su estudio hablaba mayoritariamente español. No obstante, y al margen de las evidentes diferencias de raza entre la población española y la población de Puerto Rico, como sugieren Cintrón et al., existen otras diferencias tales como la localización geográfica, las circunstancias económicas, la historia del - país y la situación política, que pueden influir en la manifestación de la ansiedad. El modelo factorial obtenido por Cintrón et al. (en prensa) posee cierta semejanza con la estructura bifactorial postulada por Sandín et al. (2002) para la SA en niños y adolescentes y, más recientemente, por Zvolensky et al. (2003) para una forma revisada de la ASI, estructura que consiste en un factor de SA somática y un factor social-cognitivo (el primero guarda cierta correspondencia con el factor de SA y el segundo con el factor emocional de Cintrón et al.).

Aunque en nuestro anterior estudio sobre la estructura de la ASI basado en una muestra de pacientes con trastornos de ansiedad obtuvimos evidencia a favor de un modelo unidimensional más que multidimensional (Sandín et al., 1996), los resultados que aquí presentamos sobre la evidencia de un factor general (en el nivel más alto de la jerarquía), así como también las elevadas correlaciones entre los tres factores primarios, son congruentes con la existencia de un constructo unitario de sensibilidad a la ansiedad. En nuestros datos, una proporción importante de la varianza en las puntuaciones de la ASI está explicada por el factor general, y una proporción significativa por el grupo de factores primarios, lo cual indica la existencia de una estructura multidimensional y a la vez de un componente general de SA. En los estudios de Zinbarg et al. (1997), Stewart et al. (1997) y Mohlman y Zinbarg (2000) se obtienen resultados que sugieren una estructura similar a la aquí presentada, así como también la existencia de un factor general que explica una proporción elevada de la varianza en las puntuaciones a la ASI. No obstante, aunque parece claro que existe un constructo general de sensibilidad a la ansiedad, y que en cierto modo estaría reflejado en la puntuación total de la ASI, lo que aún no se conoce es su naturaleza precisa (aun así, no parece probable que consista en una modalidad de emocionalidad general, sino más bien de SA general).

La existencia de un modelo jerárquico de la SA posee importantes implicacio- 
nes para la psicopatología y el tratamiento de la ansiedad. En primer lugar, el hecho de que existan tres componentes de SA que son al menos parcialmente distintos (SA-somática, SA-cognitiva, y SA-social), permite describir este constructo psicopatológico de forma diferente a como se ha venido haciendo (i.e., unidimensional o consistente en cuatro dimensiones independientes o relacionadas). Igualmente permite derivar subescalas de SA y relacionar estas dimensiones primarias con otros constructos psicólogos de la ansiedad y otras características psicopatológicas. Mientras que ciertos componentes de SA, o el factor general, pueden estar etiológicamente más implicados en unos trastornos de ansiedad que en otros, también pueden ser de gran utilidad para estudios sobre la predicción y evolución de los trastornos de ansiedad. Por ejemplo, mientras que la SA-somática parece estar más relacionada con el trastorno de pánico (Zinbarg et al., 1997; Brown, Smits, Powers y Telch, 2003), la SA-social presenta una relación más estrecha con el la fobia social (Zinbarg et al., 1997). Así mismo, se ha sugerido que la SA-cognitiva presenta una cierta asociación con la depresión mayor (Taylor, Koch, Woody y McLean, 1996). En segundo lugar, el hecho de que exista una estructura jerárquica, y en concreto un factor general de SA, podría plantear dudas sobre la conveniencia de utilizar la puntuación total de la ASI como medida del constructo general (unitario) de SA. Sin embargo, puesto que la evidencia empírica sugiere que este factor general explica una proporción importante de la varianza en las puntuaciones de la ASI, podría afirmarse que la puntuación total de la SI es una medida adecuada del constructo unitario de SA (representado por el factor general).

La ASI está traducida a muchos idiomas y, al menos hasta el momento pre- sente, es sin duda la prueba de evaluación de la SA más utilizada internacionalmente. Hasta el momento actual, la ASI se ha empleado mayoritariamente como una medida unidimensional (general) de la sensibilidad a la ansiedad. Sin embargo, la naturaleza multidimensional de esta escala sugiere la posibilidad y conveniencia de derivar al menos tres subescalas sobre los tres componentes de SA aislados (somático, cognitivo y social). Aunque las tres dimensiones resultan consistentes en general, especialmente la dimensión social parece presentar algunas inconsistencias en nuestros análisis exploratorios que deberán ser evaluadas en futuras investigaciones. Así mismo, como ya indicamos en la sección de resultados, algunos items podrian asignarse indistintamente a una u otra dimensión. Por ejemplo, el Ítem 3 ( $« \mathrm{Me}$ asusto cuando siento que tiemblo") ha sido asignado al factor cognitivo, aunque en el grupo de mujeres corresponde al factor social; este mismo ítem fue asignado por Zinbarg et al. (1997) y Stewart et al. (1997) al factor somático. Algunos autores han venido sugiriendo la necesidad de una revisión de la ASI, al menos para mejorar las dimensiones cognitiva y social (p.ej., Zinbarg et al., 1997, 1999; Mohlman y Zinbarg, 2000). Una posible solución a esta cuestión ha venido dada por la aparición de la ASI-Revisada (ASI-R; Taylor y Cox, 1998; Zvolensky et al., 2003). Esta nueva escala posee 36 items y evalúa 6 dimensiones de SA [SA-social, SA-neurológica/disociación, SA-cognitiva, y tres dimensiones de SA somática (cardiovascular, respiratoria y gastrointestinal]. Aunque esta nueva escala supone una importante mejora sobre su antecesora, es muy probable que esta última siga empleándose con elevada frecuencia debido a su brevedad y a la multitud de trabajos que han probado su consistencia, validez y utilidad clínica. 


\section{REFERENCIAS BIBLIOGRÁFICAS}

Bentler, P.M. (1995). EQS Structural equations program manual. Encino, CA: Multivariate Software, Inc.

Brown, M., Smits, J.A.J., Powers, M.B., y Telch, M.J. (2003). Differential sensitivity of the three ASI factors in predicting panic disorder patients' subjective and behavioural response to hyperventilation challenge. Journal of Anxiety Disorders, 17, 583-591.

Carter, M.M., Miller, O., Sbrocco, T., Suchday, S., y Lewis, E.L. (1999). Factor structure of the Anxiety Sensitivity Index among African American college students. Psychological Assessment, 11, 525-533.

Cattell, R.B. (1966). The scree test for the number of factors. Multivariate Behavioral Research, 3, 245-276.

Cintrón, J.A., Carter, M.M., Suchday, S., Sbrocco, T., y Gray, J. (en prensa). Factor structure and construct validity of the Anxiety Sensitivity Index among island Puerto Rico. Journal of Anxiety Disorders.

Cox, B.J., Borger, S.C., y Enns, M.W. (1999). Anxiety sensitivity and emotional disorders: Psychometric studies and their theoretical implications. En S. Taylor (Ed.), Anxiety sensitivity: Theory, research, and treatment of the fear of anxiety (pp. 115148). Mahwah, NJ: LEA.

Cox, B.J., Parker, J.A., y Swinson, R.P. (1996). Anxiety sensitivity: Confirmatory evidence for a multidimensional construct. Behaviour Research and Therapy, 34, 591-598.

Gorsuch, R. (1983). Factor analysis. Hillsdale, NJ: Erlbaum.

McNally, R.J. (1994). Panic Disorder: A critical analysis. New York: Guilford Press.

McNally, R.J. (2002). Anxiety sensitivity and panic disorder. Biological Psychiatry, 52, 938-946.

Mohlman, J., y Zinbarg, R.E. (2000). The structure and correlates of anxiety sensitivity in older adults. Psychological Assessment, 12, 440-449.

Norton, P.J., De Coteau, T.J., Hope, D.A., y Anderson, J. (2004). The factor structure of the Anxiety Sensitivity Index among northern plains native Americans. Behaviour Research and Therapy, 42, 241-147.
Peterson, R.A., y Reiss, R.J. (1992). Anxiety Sensitivity Index Manual ( ${ }^{\text {nd }}$ edition). Worthington, $\mathrm{OH}$ : International Diagnostic Systems.

Reiss, S. (1991). Expectancy model of fear, anxiety, and panic. Clinical Psychology Review, 11, 141-153.

Reiss, S., y McNally, R.J. (1985). Expectancy model of fear. En S. Reiss y R.R. Bootzin (Eds.), Theoretical issues in behavior therapy (pp. 107-122). New York: Academic Press.

Reiss, S., Peterson, R.A., Gursky, D.M., y McNally, R.J. (1986). Anxiety sensitivity, anxiety frequency, and the prediction of fearfulness. Behaviour Research and Therapy, 24, 1-8.

Rodríguez, B.F., Bruce, S.E., Pagano, M.E., Spencer, M.A., y Séller, M.B. (2004). Factor structure and stability of the Anxiety Sensitivity Index in a longitudinal study of anxiety disorder patients. Behaviour Research and Therapy, 42, 79-91.

Sandin, B., Chorot, P., y McNally, R.J. (1996). Validation of the Spanish version of the Anxiety Sensitivity Index in a clinical sample. Behaviour Research and Therapy, 34, 283-290.

Sandín, B., Chorot, P., y McNally, R.J. (2001). Anxiety sensitivity index: Normative data and its differentiation from trait anxiety. Behaviour Research and Therapy, 39, 213 219.

Sandín, B., Chorot, P., Santed, M.A., y Valiente, R.M. (2002). Análisis factorial confirmatorio del Índice de Sensibilidad a la Ansiedad para Niños. Psicothema, 14, 333-339.

Sandín, B., Valiente, R.M., y Chorot, P. (1999). Material de apoyo para la evaluación de los miedos y fobias. En B. Sandín (Ed.), Las fobias especificas (pp. 161-188). Madrid: Klinik.

Schmid, J., y Leiman, J.M. (1957). The development of hierarchical factor solutions. Psychometrica, 22, 53-61.

Schmidt, N.B, y Joiner, T.E. (2002). Structure of the Anxiety Sensitivity Index: Psychometrics and factor structure in a community sample. Journal of Anxiety Disoders, $16,33-49$.

Stewart, S.H., Taylor, S., y Baker, J.M. (1997). Gender differences in dimensions of anxiety sensitivity. Journal of Anxiety Disorders, 11, 179-200. 
Taylor, S. (1995a). Anxiety sensitivity: Theoretical perspectives and recent findings. Behaviour Research and Therapy, 33, 243-258.

Taylor, $\mathrm{S}$. (1995b). Issues in the conceptualization and measurement of anxiety sensitivity. Journal of Anxiety Disorders, 9, 163-174.

Taylor, S., y Cox, B.J. (1998). An expanded Anxiety Sensitivity Index: Evidence for a Hierarchic structure in a clinical sample. Journal of Anxiety Disorders, 12, 463-483.

Taylor, S., Koch, W.J., y Crockett, D.J. (1991). Anxiety sensitivity, trait anxiety, and the anxiety disorders. Journal of Anxiety Disorders, 5, 293-311.

Taylor, S., Koch, W.J., y McNally, R.J. (1992). How does anxiety sensitivity vary across the anxiety disorders? Journal of Anxiety Disorders, 6, 249-259.

Taylor, S., Koch, W.J., Woody, S., y McLean, P. (1996). Anxiety sensitivity and depression: How are they related? Journal of Abnormal Psychology, 105, 474-479.

Telch, M.J., Shermis, M.D., y Lucas, J.A. (1989). Anxiety sensitivity: Unitary perso- nality trait or domain-specific appraisals? Journal of Anxiety Disorders, 3, 25-32.

Valiente, R.M., Sandín, B., y Chorot, P. (2002). Miedos comunes en niños y adolescentes: Relación con la sensibilidad a la ansiedad, el rasgo de ansiedad, la afectividad negativa y la depresión. Revista de Psicopatología y Psicología Clínica, 7, 61-70.

Zinbarg, R.E., Barlow, D.H., y Brown, T.A. (1997). Hierarchical structure and general factor saturation of the Anxiety Sensitivity Index: Evidence and implications. Psychological Assessment, 9, 277-284.

Zinbarg, R.E., Mohlman, J., y Hong, N.H. (1999). Dimensions of anxiety sensitivity. En S. Taylor (Ed.), Anxiety sensitivity: Theory, research, and treatment of the fear of anxiety (pp. 83-114). Mahwah, NJ: LEA. Zvolensky, M.J., Arrindell, W.A., Taylor, S., Bouvard, M., Cox, B.J., Stewart, S.H., Sandin, B., Jurado Cardenas, S., y Eifert, G.H. (2003). Anxiety sensitivity in six countries. Behaviour Research and Therapy, 41, 841859. 


\section{Anexo I. Escala de sensibilidad a la ansiedad ASI (Anxiety Sensitivity Index)}

\section{A S I}

Nombre Edad. Sexo (varón mujer_

Conteste al siguiente cuestionario rodeando con un círculo la alternativa $(0,1,2,3,4)$ que mejor refleje el grado en que está de acuerdo con cada uno de los enunciados que se indican a continuación, teniendo en cuenta la siguiente gradación:

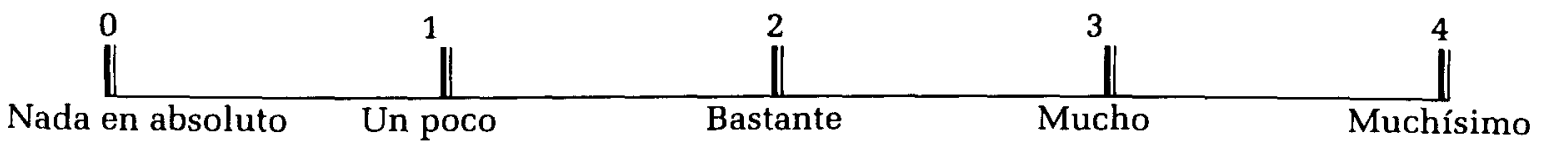

Procure contestar a todas las frases de acuerdo con su propia experiencia. No obstante, si alguna de las frases se refiere a algo que Vd. no ha vivido o experimentado nunca, conteste en base a lo que Vd. piense que podría sentir si hubiera tenido tal experiencia. Por favor conteste a todas las frases.

\begin{tabular}{|c|c|c|c|c|c|}
\hline \multirow[b]{2}{*}{ 1. Para mí es importante no dar la impresión de estar nervioso/a } & \multicolumn{3}{|c|}{$\begin{array}{c}\text { nada en } \\
\text { absoluto }\end{array}$} & \multicolumn{2}{|c|}{$\begin{array}{l}\text { muchi } \\
\text { simo }\end{array}$} \\
\hline & 0 & 1 & 2 & 3 & 4 \\
\hline $\begin{array}{l}\text { 2. Cuando no puedo mantener mi mente concentrada en una tarea, } \\
\text { siento la preocupación de que podría estar volviéndome loco/a }\end{array}$ & 0 & 1 & 2 & 3 & 4 \\
\hline 3. Me asusto cuando siento que tiemblo & 0 & 1 & 2 & 3 & 4 \\
\hline 4. Me asusto cuando siento que me desmayo o me mareo & 0 & 1 & 2 & 3 & 4 \\
\hline 5. Es importante para mí mantener el control de mis emociones & 0 & 1 & 2 & 3 & 4 \\
\hline 6. Me asusto cuando mi corazón late de forma rápida & 0 & 1 & 2 & 3 & 4 \\
\hline 7. Me resulta embarazoso o molesto cuando mi estómago hace ruidos & 0 & 1 & 2 & 3 & 4 \\
\hline 8. Me asusto cuando tengo náuseas (sensación de vómito) & 0 & 1 & 2 & 3 & 4 \\
\hline $\begin{array}{l}\text { 9. Cuando noto que mi corazón está latiendo muy deprisa, me preocupa } \\
\text { que pueda tener un ataque cardíaco }\end{array}$ & 0 & 1 & 2 & 3 & 4 \\
\hline 10. Me asusto cuando no puedo respirar hondo o respiro con dificultad & 0 & 1 & 2 & 3 & 4 \\
\hline $\begin{array}{l}\text { 11. Cuando me molesta el estómago, me preocupa que pueda estar } \\
\text { seriamente enfermo/a }\end{array}$ & 0 & 1 & 2 & 3 & 4 \\
\hline $\begin{array}{l}\text { 12. Me asusto cuando soy incapaz de mantener mi mente concentrada } \\
\text { en una tarea }\end{array}$ & 0 & 1 & 2 & 3 & 4 \\
\hline 13. Cuando siento que tiemblo la gente suele darse cuenta & 0 & 1 & 2 & 3 & 4 \\
\hline 14. Las sensaciones corporales poco habituales (raras) me asustan & 0 & 1 & 2 & 3 & 4 \\
\hline $\begin{array}{l}\text { 15. Cuando estoy nervioso/a me preocupa que pueda estar mentalmente } \\
\text { enfermo/a }\end{array}$ & 0 & 1 & 2 & 3 & 4 \\
\hline 16. Me asusto cuando estoy nervioso/a & 0 & 1 & 2 & 3 & 4 \\
\hline
\end{tabular}

(C) 1992 R. A. Peterson y S. Reiss. Versión española B. Sandín y P. Chorot (publicado en: Sandin, B., Valiente, R.M., y Chorot, P. (1999). Material de apoyo para la evaluación de los miedos y las fobias. En B. Sandín (Ed.), Las fobias específicas. Madrid: Klinik, pp. 179-180]. Reproducido con permiso. 